\title{
Numerical approaches to time evolution of complex quantum systems
}

\author{
Holger Fehske $^{\mathrm{a}}$, Jens Schleede ${ }^{\mathrm{a}}$, Gerald Schubert ${ }^{\mathrm{a}, \mathrm{b}}$, Gerhard Wellein $^{\mathrm{b}}$, Vladimir S. Filinov ${ }^{\mathrm{c}}$, Alan R. Bishop ${ }^{\mathrm{d}}$ \\ ${ }^{a}$ Institut für Physik, Ernst-Moritz-Arndt Universität Greifswald, Felix-Hausdorff-Str. 6, 17487 Greifswald, Germany \\ ${ }^{b}$ Regionales Rechenzentrum Erlangen, Friedrich-Alexander-Universität Erlangen-Nürnberg, Martensstr.1, 91058 Erlangen, Germany \\ ${ }^{c}$ Joint Institute for High Temperatures, Russian Academy of Sciences, Moscow 127412, Russia \\ ${ }^{d}$ Theory, Simulation and Computation Directorate, Los Alamos National Laboratory, Los Alamos, New Mexico 87545, U.S.A.
}

\begin{abstract}
We examine several numerical techniques for the calculation of the dynamics of quantum systems. In particular, we 'single out an iterative method which is based on expanding the time evolution operator into a finite series of Chebyshev polynomials. The Chebyshev approach benefits from two advantages over the standard time-integration Crank-Nicholson 'scheme: speedup and efficiency. Potential competitors are semiclassical methods such as the Wigner-Moyal or quantum tomographic approaches. We outline the basic concepts of these techniques and benchmark their performance against the Chebyshev approach by monitoring the time evolution of a Gaussian wave packet in restricted one-dimensional (1D) geometries. Thereby the focus is on tunnelling processes and the motion in anharmonic potentials. Finally we apply the 'prominent Chebyshev technique to two highly non-trivial problems of current interest: (i) the injection of a particle in a disordered 2D graphene nanoribbon and (ii) the spatiotemporal evolution of polaron states in finite quantum systems. Here, depending on the disorder/electron-phonon coupling strength and the device dimensions, we observe transmission or localisation of the matter wave.
\end{abstract}

\section{Introduction}

Quantum statistical physics, such as condensed matter or plasma physics, but also quantum chemistry, heavily 'depends on effective numerical methods for solving complex few-particle and many particle problems. Implementing suitable theoretical concepts for their description on modern supercomputer architectures, nowadays computational physics constitutes, besides experiment and theory, the third pillar of contemporary physics [8]. Numerical techniques become especially important for strongly correlated systems where analytical approaches largely fail. This is due to the absence of small (coupling) parameters or, more general, because the relevant energy scales are not well separated, both preventing the application of standard perturbative schemes.

Common to any numerical method in quantum physics is the requirement to represent the states and operators 'describing the physical system in the Hilbert space in a form that is suited for computations. Then, working with a discrete basis of the Hilbert space, the computational challenge is the solution of an eigenvalue problem for huge (sparse) matrices. For most physical systems the dimension of the Hilbert space is much too large in order to perform a full exact diagonalisation of the related Hamilton matrix. Fortunately some quantities of interest depend on the properties of the ground state or a few excited states only, and therefore may be studied by iterative Krylov space techniques such as Lanczos diagonalisation [5]. The quantum dynamics or long time behaviour of correlated systems, however, require, in principle, the knowledge of all eigenstates.

The theoretical investigation of quantum dynamics was triggered in recent years by the vast progress of the experimental techniques. Nowadays femtosecond laser spectroscopy, for instance, allows for a precise analysis of quantum dynamical processes with extreme time resolution. Direct time integration of the Schrödinger equation at the cost of a full diagonalisation of the system's Hamiltonian (including the coupling to external fields) is impractical in such cases because of its computational complexity.

The aim of this work is to propose a very efficient Chebyshev-based algorithm that allows calculating the dynamics of a quantum system numerically exactly, also for relatively long times, and therefore overcomes the above mentioned problem at least partially. In order to demonstrate the power of our iterative Chebyshev expansion approach, we compare the accuracy and computational costs of certain model calculations with those emerging by the use of the more the standard Crank-Nicholson, WignerMoyal and quantum tomography methods. We start by presenting the basic ideas of the iterative (Chebyshev, CrankNicholson; Sect. 2.1) and semiclassical (Wigner-Moyal, tomographic; Sect. 2.3) techniques. Afterwards, in Sect. 3 , we consider three different problems of increasing complexity: (i) the motion of a Gaussian wave packet in a $1 \mathrm{D}$ geometry (Sec. 3.1), (ii) the evolution of a particle in a disordered 2D graphene nanoribbon (Sec. 3.2), and (iii) the spatiotemporal evolution of polaron states in finite quantum systems (Sec. 3.3). Our conclusions will be presented in Sect. 4 . 


\section{Time evolution of quantum systems}

The time evolution of a quantum state $|\psi\rangle$ is described by the Schrödinger equation

$$
\mathrm{i} \hbar \frac{\partial}{\partial t}|\psi(t)\rangle=H|\psi(t)\rangle .
$$

If the Hamilton operator $H$ does not explicitly depend on time $t$ we can formally integrate this equation and express the dynamics of a given quantum state $\left|\psi\left(t_{0}\right)\right\rangle$ in terms of the time evolution operator $U\left(t, t_{0}\right)$ as $|\psi(t)\rangle=$ $U\left(t, t_{0}\right)\left|\psi\left(t_{0}\right)\right\rangle$, where $U\left(t, t_{0}\right)=e^{-\mathrm{i} H\left(t-t_{0}\right) / \hbar}$. Exploiting that $U\left(t, t_{0}\right)$ is diagonal in the eigenbasis of the Hamiltonian, we can directly determine the dynamics of the quantum system.

\subsection{Direct method}

For systems with moderate Hilbert space dimensions, a full diagonalisation of the Hamiltonian permits expression of the quantum dynamics of an initial state $\left|\psi\left(t_{0}\right)\right\rangle$ as

$$
|\psi(t)\rangle=\sum_{n=1}^{N} e^{-\mathrm{i} E_{n}\left(t-t_{0}\right) / \hbar}|n\rangle\left\langle n \mid \psi\left(t_{0}\right)\right\rangle .
$$

Here $|n\rangle$ are the (time independent) eigenstates of the system and $E_{n}$ the corresponding eigenenergies. In this way, the decomposition of an initial state into a linear combination of eigenstates allows for an exact calculation of the quantum state at arbitrary times. As soon as the physical description of the system requires a larger Hilbert space dimension, however, this direct calculation is no longer feasible and we have to resort to alternative approaches.

\subsection{Iterative methods}

Crank-Nicholson scheme. One of the standard methods to calculate the quantum time evolution iteratively is the Crank-Nicholson algorithm [18]. Dividing $\left[t_{0}, t\right]$ into $S$ subintervals $\delta t$, the quantum state evolves for each iterative time step $\delta t$ from $t_{s}$ to $t_{s+1}=t_{s}+\delta t$ according to

$$
\left(1+\frac{1}{2} \mathrm{i} H \delta t / \hbar\right)\left|\psi\left(t_{s+1}\right)\right\rangle=\left(1-\frac{1}{2} \mathrm{i} H \delta t / \hbar\right)\left|\psi\left(t_{s}\right)\right\rangle .
$$

There are two limitations to this scheme. First, in addition to the matrix vector multiplication (MVM) on the right hand side of (3), each iteration requires the solution of a linear system of equations to obtain $\left|\psi\left(t_{s+1}\right)\right\rangle$. Despite the availability of many powerful methods for the solution of large (sparse) linear equation systems, this task remains the most time consuming part of the algorithm. Using iterative methods for the solution of the linear equation system, the attainable Hilbert space dimensions increase substantially as compared to direct methods. Second, the Crank-Nicholson algorithm is accurate only to order $(\delta t)^{2}$, which severely restricts the maximum usable iterative time step.
Chebyshev scheme. Both limitations can be overcome by an approach where we expand the time evolution operator $U\left(t, t_{0}\right)=U\left(t-t_{0}\right)=U(\Delta t)$ into a finite series of first-kind Chebyshev polynomials of order $k: T_{k}(x)=$ $\cos (k \arccos (x))$. We then obtain [4, 20, 21]

$$
U(\Delta t)=e^{-\mathrm{i} b \Delta t / \hbar}\left[c_{0}(a \Delta t / \hbar)+2 \sum_{k=1}^{M} c_{k}(a \Delta t / \hbar) T_{k}(\tilde{H})\right] .
$$

Prior to the expansion the Hamiltonian has to be shifted and rescaled such that the spectrum of $\tilde{H}=(H-b) / a$ is within the definition interval of the Chebyshev polynomials, $[-1,1]$. The parameters $a$ and $b$ are calculated from the extreme eigenvalues of $H$ as $b=\frac{1}{2}\left(E_{\max }+E_{\min }\right)$ and $a=\frac{1}{2}\left(E_{\max }-E_{\min }+\epsilon\right)$. Here we introduced $\epsilon=\alpha\left(E_{\max }-\right.$ $\left.E_{\min }\right)$ to ensure the rescaled spectrum $|\tilde{E}| \leq 1 /(1+\alpha)$ lies well inside $[-1,1]$. In practice, we use $\alpha=0.01$. Note that the Chebyshev expansion also applies to systems with unbounded spectra. In those cases we truncate the infinite Hilbert space to a finite dimension by restricting the model on a discrete space grid or using an energy cutoff. In this way we ensure the finiteness of the extreme eigenvalues.

In (4), the expansion coefficients $c_{k}$ are given by

$$
c_{k}(a \Delta t / \hbar)=\int_{-1}^{1} \frac{T_{k}(x) e^{-\mathrm{i} x a \Delta t / \hbar}}{\pi \sqrt{1-x^{2}}} d x=(-\mathrm{i})^{k} J_{k}(a \Delta t / \hbar)
$$

( $J_{k}$ denotes the $k$-th order Bessel function of the first kind).

To calculate the evolution of a state $\left|\psi\left(t_{0}\right)\right\rangle$ from one time grid point to the adjacent one, $|\psi(t)\rangle=U(\Delta t)\left|\psi\left(t_{0}\right)\right\rangle$, we have to accumulate the $c_{k}$-weighted vectors $\left|v_{k}\right\rangle=$ $T_{k}(\tilde{H})\left|\psi\left(t_{0}\right)\right\rangle$. Since the coefficients $c_{k}(a \Delta t / \hbar)$ depend on the time step but not on time explicitly, we need to calculate them only once. The vectors $\left|v_{k}\right\rangle$ can be computed iteratively exploiting the recurrence relation of the Chebyshev polynomials,

$$
\left|v_{k+1}\right\rangle=2 \tilde{H}\left|v_{k}\right\rangle-\left|v_{k-1}\right\rangle
$$

with $\left|v_{1}\right\rangle=\tilde{H}\left|v_{0}\right\rangle$ and $\left|v_{0}\right\rangle=\left|\psi\left(t_{0}\right)\right\rangle$. Evolving the wave function from one time step to the next requires $M$ MVMs of a given complex vector with the (sparse) Hamilton matrix of dimension $N$ and the summation of the resulting vectors after an appropriate rescaling. The Chebyshev expansion may also be applied to systems with time dependent Hamiltonians, but there the time variation $H(t)$ determines the maximum $\Delta t$ by which the system may be propagated in a single time step. For time independent $H$, in principle, arbitrary large time steps are possible at the expense of increasing $M$. We may choose $M$ such that for $k>M$ the modulus of all expansion coefficients $\left|c_{k}(a \Delta t / \hbar)\right| \sim J_{k}(a \Delta t / \hbar)$ is smaller than a desired accuracy cutoff. This is facilitated by the fast asymptotic decay of the Bessel functions,

$$
J_{k}(a \Delta t / \hbar) \sim \frac{1}{\sqrt{2 \pi k}}\left(\frac{e a \Delta t}{2 \hbar k}\right)^{k} \quad \text { for } \quad k \rightarrow \infty .
$$


Thus, for large $M$, the Chebyshev expansion can be considered as quasi-exact, and permits a considerably larger time step than e.g. the Crank-Nicholson scheme. Besides the high accuracy of the method, the linear scaling of computation time with both time step and Hilbert space dimension are promising in view of potential applications to more complex systems. In our cases almost all computation time is spent in sparse MVMs, which can be efficiently parallelised, allowing for a good speedup on parallel computers.

\subsection{Semiclassical methods}

During the last decades, a variety of semiclassical methods have been tailored in order to incorporate certain quantum effects at least partially into classical many-particle simulations. Based on the real time (Feynman-) path integral formulation of quantum mechanics, where action integrals take the center stage, they allow propagation of the (complex) wave function of a quantum system in time. Within the numerical evaluation of the integrals occurring by Monte Carlo (MC) techniques [10], the oscillatory complex valued integrand causes a dynamical sign problem which spoils the efficiency of the MC integration.

Wigner-Moyal approach. Since a quantum system can be described equivalently in terms of real valued quantum phase space distribution functions [15], e.g., the Wigner function, the dynamical sign problem may be alleviated [11, 12. 1. Starting from the von Neumann equation we may derive an evolution equation for the Wigner function $W(q, p, t)$

$$
\frac{\partial W}{\partial t}+\frac{p}{m} \frac{\partial W}{\partial q}+F(q) \frac{\partial W}{\partial p}=\int_{-\infty}^{\infty} d s W(q, p-s, t) \omega(s, q)
$$

where $F(q)=-\frac{d V(q)}{d q}$ is the classical force, and

$$
\omega(s, q)=\frac{2}{\pi \hbar^{2}} \int d q^{\prime} V\left(q-q^{\prime}\right) \sin \left(\frac{2 s q^{\prime}}{\hbar}\right)+F(q) \frac{d \delta(s)}{d s} .
$$

In the classical limit, the right hand side of (8) vanishes, leaving us with the Liouville equation for the phase space density. Then the dynamics can be expressed in terms of the classical propagator

$$
\begin{aligned}
\Pi^{W}\left(q, p, t ; q_{0}, p_{0}, t_{0}\right)= & \delta\left[q-\bar{q}\left(t ; p_{0}, q_{0}, t_{0}\right)\right] \\
& \times \delta\left[p-\bar{p}\left(t ; p_{0}, q_{0}, t_{0}\right)\right],
\end{aligned}
$$

where $\bar{p}$ and $\bar{q}$ are the momentum and coordinate of a trajectory that evolves according to the Hamilton equations of motion with initial conditions $\bar{p}\left(t_{0}\right)=p_{0}$ and $\bar{q}\left(t_{0}\right)=q_{0}$.

\footnotetext{
${ }^{1}$ Note that the Wigner function is just a convenient mathematical tool for the description of quantum systems and cannot be considered as a joint probability due to its possibly negative values, and conflict with Heisenberg uncertainty relation.
}

Using $\Pi^{W}$, we may rewrite (8) in form of an integral equation [11, 12],

$$
\begin{aligned}
W(q, p, t)= & \int d p_{0} d q_{0} \Pi^{W}\left(q, p, t ; q_{0}, p_{0}, t_{0}\right) W_{0}\left(q_{0}, p_{0}, t_{0}\right) \\
& +\int_{t_{0}}^{t} d \tau \int d p_{\tau} d q_{\tau} \Pi^{W}\left(q, p, t ; q_{\tau}, p_{\tau}, \tau\right) \\
& \times \int_{-\infty}^{\infty} d s W\left(q_{\tau}, p_{\tau}-s, \tau\right) \omega\left(s, q_{\tau}\right),
\end{aligned}
$$

and solve it by iteration. Here, we consider only the lowest order, which means that the second integral is neglected completely. That is, we propagate classical trajectories $(\bar{q}, \bar{p})$ in time, after sampling their initial conditions $p_{0}$ and $q_{0}$ from the initial Wigner function $W_{0}\left(q_{0}, p_{0}, t_{0}\right)$ at time $t_{0}$ by a MC procedure. Their superposition at the next time grid point gives $W(q, p, t)$. The importance of higher order terms in the iteration series was investigated in Refs. 11, 12.

Quantum tomographic approach. As the dynamical sign problem is still present for the Wigner function, some years ago the description of quantum states in terms of a strictly positive function, the so called quantum tomogram, has been proposed [16]. Such a description seems promising in view of an effective MC sampling of the tra11] ectories during the propagation. The quantum tomogram [2, 3],

$$
\tilde{w}(X, \mu, \nu, t)=\int \frac{d k d q d p}{2 \pi} W(q, p, t) e^{-\mathrm{i} k(X-\mu q-\nu p)},
$$

relates to the Wigner function by a class of Radon transforms [6] which are characterised by $\mu$ and $\nu$. Each tomogram contains a density information, and tuning $(\mu, \nu)$ appropriately, we may continuously switch between coordinate and momentum representation. Also for the quantum tomogram, an evolution equation can be derived from the von Neumann equation

$$
\begin{array}{r}
\frac{\partial \tilde{w}}{\partial t}-\frac{\mu}{m} \frac{\partial \tilde{w}}{\partial \nu}-\frac{\mathrm{i}}{\hbar}\left[V\left(-\frac{\partial}{\partial \mu} \frac{1}{\partial / \partial X}-\frac{\mathrm{i} \hbar \nu}{2} \frac{\partial}{\partial X}\right)\right. \\
\left.-V\left(-\frac{\partial}{\partial \mu} \frac{1}{\partial / \partial X}+\frac{\mathrm{i} \hbar \nu}{2} \frac{\partial}{\partial X}\right)\right] \tilde{w}=0 .
\end{array}
$$

For harmonic potentials, Eq. (13) can be reformulated as a continuity equation and solved by trajectory methods. For potentials of arbitrary shape, the complicated structure prevents a direct evaluation of (13) in order to get $\tilde{w}(X, \mu, \nu, t)$. A possible way out is a local expansion of the potential up to second order [19]. The identification with the continuity equation then holds locally for each coordinate about which the potential is expanded. Since the slope and local curvature of the potential enter the propagator for the trajectories, the efficiency of the 
tomogram-reconstruction depends crucially on the choice of the potential sampling positions. An intuitive, albeit not unique, choice for those positions are the coordinates of classically evolving particles similar to those in the Wigner approach. Advantageously, it is not necessary to propagate the whole set of tomograms if one is interested in the tomogram in one reference frame $(\mu, \nu)$ only. Instead, it is sufficient to find those trajectories which end up in a given $(X(t), \mu(t), \nu(t))$, or equivalently to propagate the desired $(X, \mu, \nu)$-trajectories backward in time: one needs to be aware of the non-uniqueness of the propagator due to the various possible choices of the potential sampling points.

\section{Topical applications in physics}

We now apply the numerical techniques presented in the preceding section to selected physical systems and situations. As a first step, we calibrate the different approaches by studying a simple double well toy model. Detecting the limitations and prospects of the various methods seems to be necessary before applying them to the more complicated problems of current interest. Let us point out that the considered implementations of the semiclassical approaches provide an approximate description of quantum mechanics only, i.e. they will clearly not reproduce all the quantum effects. For the finite graphene nanoribbons studied in the second example it is barely possible to determine the full quantum dynamics by means of direct diagonalisation techniques or applying the CrankNicholson scheme. This gives us the opportunity to benchmark their performance in comparison to the Chebyshev expansion for a system for which the Hamilton matrix is not tridiagonal. The last example has been chosen to demonstrate the applicability of the Chebyshev approach to a true many-particle problem, the tunnelling of a polaron. There the Hilbert space dimension is so large that neither the direct diagonalisation method nor the CrankNicholson can be applied.

\subsection{Double well potential}

As a basic test case we consider the motion of a Gaussian wave packet in the $1 \mathrm{D}$ double well potential

$$
V(q)=V_{0}+\frac{1}{2} m \omega_{0}^{2}\left(-q^{2}+a q^{4}\right)
$$

sketched in the top panel of Fig. 11 We use $m=u_{m}$, $\omega_{0} u_{t}=0.4, a u_{\ell}^{2}=0.02$ and $V_{0}=u_{E}=u_{m} u_{\ell}^{2} / u_{t}^{2}$, where $u_{m}, u_{t}, u_{\ell}$ are the reference units for mass, time and length, respectively. The initial Gaussian of width $\sigma$ is centred at $q_{0}$, with centre-of-mass momentum $p_{0}$,

$$
\psi\left(q, t_{0}\right)=\frac{1}{\left(2 \pi \sigma^{2}\right)^{1 / 4}} \exp \left\{-\frac{1}{4 \sigma^{2}}\left(q-q_{0}\right)^{2}+\frac{\mathrm{i}}{\hbar} p_{0} q\right\},
$$

where we choose $p_{0}=u_{m} u_{\ell} / u_{t}, q_{0}=-5 u_{\ell}$, and $\sigma=$ $u_{\ell} / \sqrt{2}$. The top panel of Fig. 1 gives also the real and
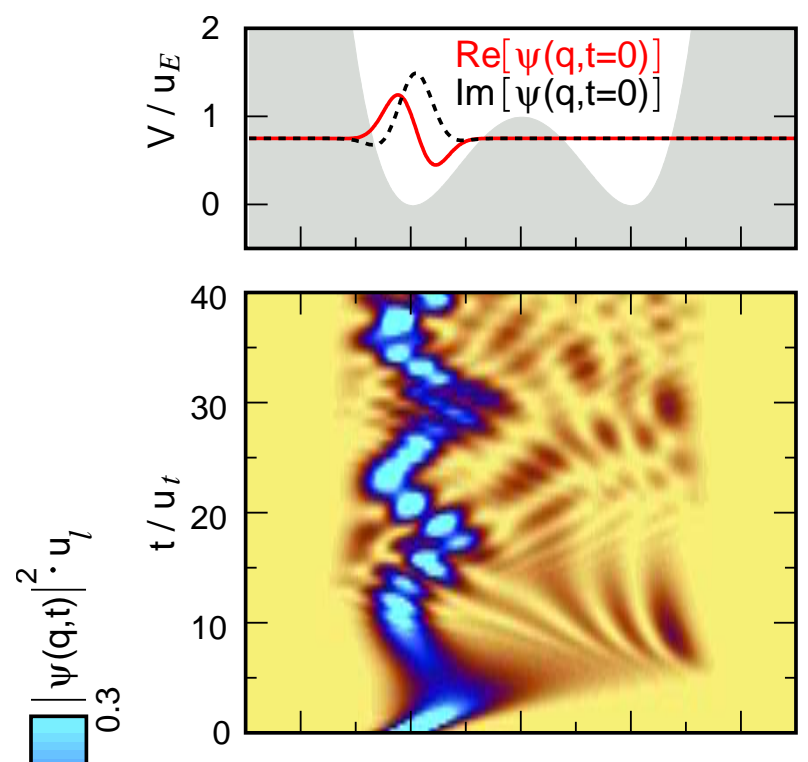

$-{ }^{1}=$
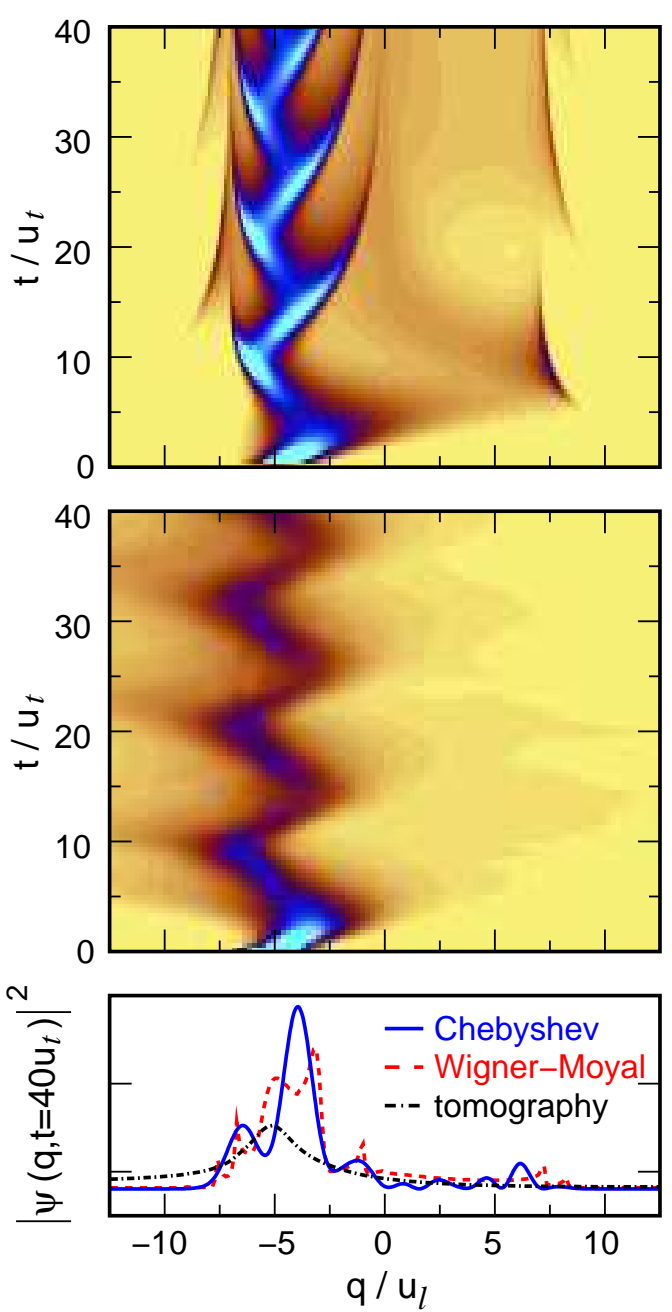

Figure 1: Time evolution of a Gaussian wave packet in a double well potential (top panel). Results displayed are obtained using the Chebyshev, the first order Wigner-Moyal and the tomographic approach (from top to bottom), respectively. 
imaginary part of the wave function, where the baseline indicates the energy of the initial state in relation to $V(q)$.

Discretising the potential $V(q)$ on an equally spaced grid of $N=2048 q$-points, we fully diagonalise the Hamiltonian to get the exact dynamics as reference. If we choose the iterative time step accordingly, the real and imaginary part of the wave function at $t=40 u_{t}$ is reproduced by both iterative methods with an absolute error less than $10^{-9}$. For this the maximum allowable time step for the CrankNicholson scheme is $\delta t=4 \times 10^{-6} u_{t}$. For the Chebyshev approach, the accuracy is even better than $2 \times 10^{-11}$. Here the required time step is related to the order of the Chebyshev expansion, i.e. the number of moments $M$. For $M=1500$ we may propagate the wave function by $\Delta t=0.4 u_{t}$. At the expense of calculating a larger number of moments $M$, larger times steps may be chosen without loss of accuracy. This will be demonstrated in the next section. The time evolution of the modulus squared of the wave function is shown in the second panel of Fig. 1. While the major part of the wave packet stays in the left well, a sizeable fraction also penetrates the barrier. The rich structure in the density pattern reflects the (well-known) presence of strong interference effects.

Restricting the Wigner-Moyal scheme to first order, the corresponding data reproduces in essence the overall dynamics, but the fine interference patterns are not resolved within this approximation (see third panel of Fig.11). There are two major parameters which influence the computation time for this approach: (a) the number of propagated trajectories and (b) the time step necessary for their classical propagation. For the results presented, we have used $5 \times 10^{5}$ trajectories and a propagation time step of $0.04 u_{t}$. The agreement between the exact solution and the tomographic result (see also the bottom panel of Fig. 1) is even worse as the tunnelling to the right hand side of the barrier is missed almost completely. Furthermore, the probability density for large negative values is overestimated, i.e. the effect of the steep anharmonic confinement potential is not accounted for correctly. In Tab. 1 we summarise the run times $T_{\text {run }}$ on a single Xeon 5160 processor required by the different methods in order to follow the time evolution of the system up to $t=40 t_{0}$.

This very basic example already shows that a straightforward use of the Wigner-Moyal and tomographic approaches only partially accounts for quantum effects. While, in principle, both methods are equivalent with respect to the solution of the time dependent Schrödinger equation, an efficient implementation is lacking. For the WignerMoyal formalism there are two prospects. If the accuracy of the first order approximation is satisfactory, i.e. the neglect of the fine interference patterns is tolerable, this method provides an acceptable performance and might show its true virtue for many-particle systems. If one has to include higher terms of the iteration series, however, e.g. because subtle quantum effects are important, the method is not competitive anymore because (i) the computational requirements increase drastically and (ii)

\begin{tabular}{|c||c|c|c|c|c|}
\hline & ED & CN & C & WM & T \\
\hline$T_{\text {run }}[s]$ & 13.8 & 1871 & 4.8 & 13.6 & 579 \\
\hline
\end{tabular}

Table 1: Time evolution of a wave packet in the double well potential up to $t=40 t_{0}$. Data gives the run times by exact diagonalisation (ED), Crank-Nicholson (CN), Chebyshev (C), Wigner-Moyal (WM) and tomographic $(\mathrm{T})$ methods.

numerical fluctuations amplify strongly during the calculation [19]. The practical applicability of the tomographic approach to arbitrarily shaped potentials is also questionable, mainly because there is no simple way to construct suitable sampling functions for the coordinate sampling in the potential evaluation. Extensions beyond harmonic potentials will suffer crucially from this limitation. Apart from the poor accuracy of the results, also the computational requirements were significant higher than for the other methods (although we used only 800 trajectories in this calculation).

\subsection{Disordered graphene nanoribbons}

Recently much interest has been devoted to investigate how disorder influences the transport properties of graphene [17, 23]. It is known that the presence of arbitrarily weak disorder leads to Anderson localisation of the single particle wave function on infinite $2 \mathrm{D}$ square lattices [1]. In weakly disordered 2D systems the localisation lengths are huge, however, and may easily become comparable to the system sizes that are technologically relevant e.g. for graphene nanoribbons. In those cases, we expect a conducting behaviour of the device despite the presence of disorder causing localisation on larger length scales.

To investigate the influence of Anderson disorder for finite graphene nanoribbons, we consider a tight-binding model on a honeycomb lattice,

$$
H=-\bar{t} \sum_{\langle i j\rangle}\left(c_{i}^{\dagger} c_{j}+\text { H.c. }\right)+\sum_{j=1}^{N} \epsilon_{j} c_{j}^{\dagger} c_{j} .
$$

Here the operators $c_{j}^{\dagger}\left(c_{j}\right)$ create (annihilate) an electron in a Wannier state centred at site $j$. The on-site potentials $\epsilon_{j}$ are random variables in the interval $[-W / 2, W / 2]$, where $W$ is a measure for the disorder strength. The electron transfer between nearest-neighbour lattice sites $\langle i j\rangle$ is described by the transfer integral $\bar{t}$. Tailoring stripes (ribbons) out of the infinite honeycomb-lattice, we have to distinguish two cases with respect to the boundary conditions. Depending on the orientation of the stripe we get boundaries of either zigzag or armchair type. Since in experimental probes armchair edges are more common, we will focus on those in the following.

Starting from a wave packet which is localised on one site in the centre of each ribbon, we evolve the quantum state using the Chebyshev approach described in Sect. 2.2. We consider devices of $1.11 \times 212.8 \mathrm{~nm}^{2}$ with $10 \times 1000$ atoms. The main panel of Fig. 2 displays the time evolution of the wave packet for one realization of disorder 


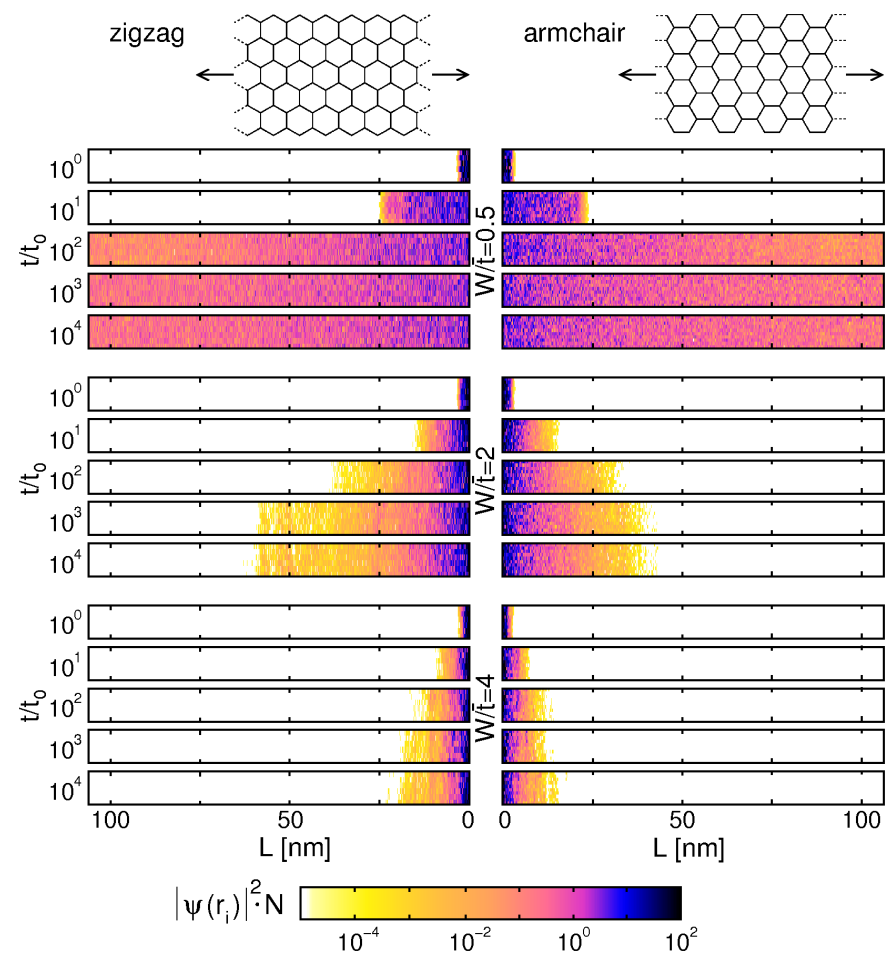

Figure 2: Time evolution of a localised state on an armchair graphene nanoribbon for different values of disorder. The left/right asymmetry of the state is due to the particular disorder realisation and initial state.

for several values of disorder strength (time is measured in units of the inverse hopping element, $\left.t_{0}=1 / \bar{t}\right)$.

The following aspects of the wave function dynamics should be noted: (i) The initially localised wave function spreads with time and reaches its maximum extension at about $t \simeq 10^{3} t_{0}$, independent of the disorder strength. Also for much longer times $\left(t \sim 10^{4} t_{0}\right)$ this extension does not change significantly anymore. (ii) The disorder strength strongly influences the spatial region over which the state spreads, i.e. the localisation length. While we see clear evidence for localisation at large disorder $(W=4 \bar{t})$, the localisation length for weak disorder $(W=0.5 \bar{t})$ is markedly larger than the system size, leading to an evenly spread state on the ribbon. Note that the occurrence of an extended (conducting) state in the latter case is only due to the finite system size. For longer ribbons a disorder of $W=0.5 \bar{t}$ is sufficient to localise the wave function as well.

The disordered nanoribbon setup gives us a good opportunity to benchmark the Chebyshev approach against the Crank-Nicholson scheme. Since the Hamiltonian is no longer tridiagonal the solution of the linear equation system cannot be done by the Thomas algorithm. Instead, we use the standard solver for double-complex linear equation systems from LAPACK, ZGESV. Table 2 summarises the number of moments required to get agreement between the Chebyshev and the exact results for different time steps $\Delta t$. The computation times for calculating the quantum state at time $t=10^{4} t_{0}$ using the various $\Delta t$ is also given.

\begin{tabular}{|c||c|c|c||c||c|}
\hline \multicolumn{1}{|c||}{} & \multicolumn{3}{c||}{$\mathrm{C}$} & $\mathrm{CN}$ & ED \\
\hline$\Delta t / t_{0}$ & 1 & 10 & 100 & $10^{-3}$ & $\infty$ \\
\hline$M$ & 104 & 264 & 1210 & - & - \\
\hline$T_{\text {run }}[s]$ & 378 & 97 & 45 & $>10^{7}$ & 1278 \\
\hline
\end{tabular}

Table 2: Numeber of Chebyshev moments (M) and overall runtime $\left(\Delta t / t_{0}\right)$ required for the calculation of the time evolution up to $t=$ $10^{4} t_{0}$ on a single Intel Xeon 5160 core.

For comparison, we also give run time $T_{\text {run }}$ for the exact diagonalisation. As one iteration of the Crank-Nicholson scheme using ZGESV takes 4 minutes, the given $T_{\text {run }}$ is only an estimate.

\subsection{Spatiotemporal evolution of polaron states in finite quantum structures}

The cycle of quasiparticle formation is fundamental to many fields of physics. In condensed matter, e.g., the coupling between a charge carrier and the lattice degrees of freedom may create a new quasiparticle, an electron dressed by an phonon cloud. This composite entity is called polaron. From the basic electron-phonon (EP) interaction processes, the absorption/emission of a phonon with a simultaneous change of the electron state, it is clear that the motion of even a single electron in a deformable lattice constitutes a complex many-body problem, in that phonons are excited at various positions, with highly nontrivial dynamics [14]. Polaron transport through finite quantum systems becomes increasingly important for nanotechnology applications.

The microscopic structure of polarons is very rich. Focusing on polaron formation in systems with short-range non-polar EP interaction and site-dependent potentials, we consider a generalised Holstein Hamiltonian [9]

$$
\begin{aligned}
H= & \sum_{i} \Delta_{i} n_{i}-\bar{t} \sum_{i}\left(c_{i}^{\dagger} c_{i+1}+\text { H.c. }\right) \\
& -\sum_{i, \sigma} \bar{g}_{i} \omega_{0}\left(b_{i}^{\dagger}+b_{i}\right) n_{i \sigma}+\omega_{0} \sum_{i} b_{i}^{\dagger} b_{i},
\end{aligned}
$$

where $c_{i}^{\dagger}\left(b_{i}^{\dagger}\right)$ creates an electron (phonon) at site $i$ of a 1D lattice. Parameters are the electron transfer integral $\bar{t}$, the EP coupling strength $\bar{g}_{i}=\left[\left(\varepsilon_{p}+\varepsilon_{p, i}\right) / \omega_{0}\right]^{1 / 2}$, and the phonon frequency $\omega_{0}$. The potentials $\Delta_{i}$ can describe a tunnel barrier, disorder, or a voltage basis.

How does a bare electron time evolve to become a polaron quasiparticle? To what extent can a polaron tunnel through a quantum barrier? Having the iterative Chebyshevbased time evolution algorithm explained in Sec. 2.2 at hand, we can address these questions in the framework of model (17). Let us emphasise that our numerical approach, acting in the tensorial product Hilbert space of electron and phonons, takes into account the full dynamics of both quantum objects. Since the Hilbert space associated to the phonons is infinite, we applied a controlled truncation procedure retaining only basis states with at most $N_{\text {ph }}$ phonons [13, 22]. However the truncated Hilbert 
space dimension $\left(D^{t o t}\right)$ is still very large even for small lattices and the dimension of the corresponding sparse matrix problem does limit the physical parameter region attainable. Thus, we use a memory saving implementation of the sparse MVM where the non-zero matrix elements are not stored but recomputed in each sparse MVM step, limiting the overall memory consumption of our implementation to five vectors of size $D^{\text {tot }}$. In this context we can access a massively parallel sparse MVM code which has proven to be sufficient to compute the ground state of the model (17) up to $D^{\text {tot }}=3.5 \times 10^{11}$ very efficiently on more than 5000 processor cores [7]. For the single polaron dynamics presented here, the matrix dimension is $D^{\text {tot }}=6.2 \times 10^{8}$ and we run the Chebyshev approach on 18 processors of a SGI Altix4700 compute server accessing a total of approximately 60 GBytes of main memory and consuming less than $500 \mathrm{CPU}$-hrs to compute the result presented in Fig. 3 .

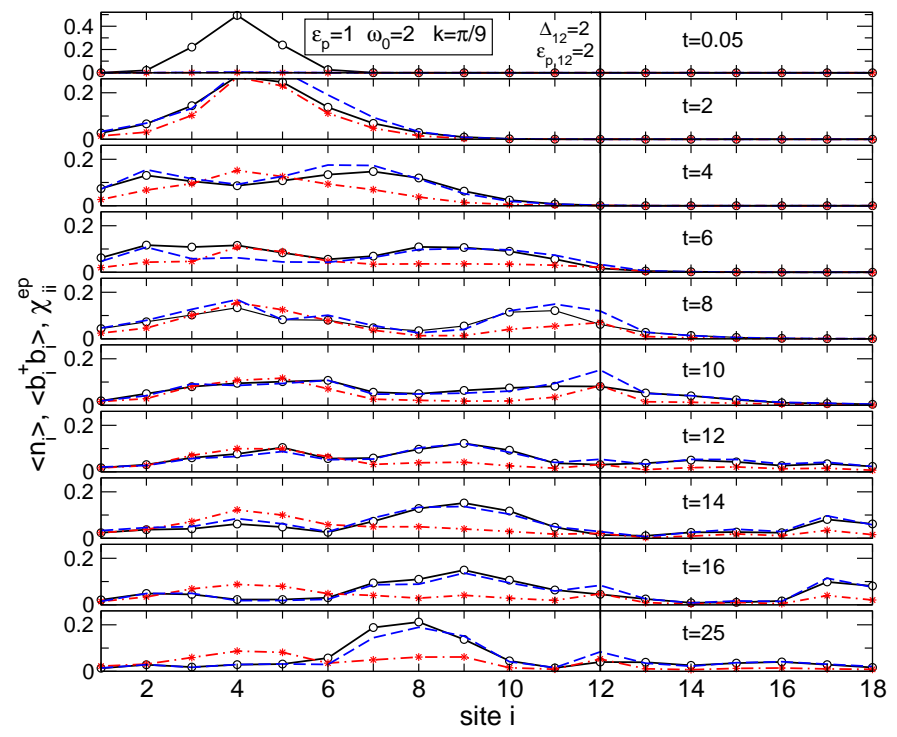

Figure 3: Quantum dynamics of polaron formation and polaron tunnelling through a potential barrier. A Gaussian wave packet centred around site 4 begins evolving at time $t=0$ with momentum $k=\pi / 9$. Moving to the right, a polaron is formed that hits the barrier located at site 12 at about $t \simeq 10$. There complicated reflection and transmission processes of the composite quasiparticle made up of an electron and phonons take place. Displayed is the timeevolution of the local particle densities $\left\langle n_{i}\right\rangle$ (solid lines, open circles), phonon numbers $\left\langle b_{i}^{\dagger} b_{i}\right\rangle$ (dot-dashed lines, stars) and EP correlations $\chi_{i i}^{e p}=\left\langle n_{i}\left(b_{i}+b_{i}^{+}\right)\right\rangle$(dashed lines). Open boundary conditions were used at sites 1 and 18. In the numerics we account for all states with up to $N_{\mathrm{ph}} \leq 11$ phonons and in the ground state the weight of all basis states containing exactly $N_{\mathrm{ph}}=11$ is less than $10^{-11}$.

Figure 3 shows snapshots of polaron formation and polaron propagation at intermediate EP coupling $\varepsilon_{p}=1$ in the non-adiabatic regime $\omega_{0}=2$ (the time and all energies were measured in units of $\bar{t}^{-1}$ and $\bar{t}$, respectively). At $t=0$ a bare electron wave packet is injected at site 4 and launched to the right. Shortly after, the electron is not yet dressed and moves nearly as fast as a free particle. But then the electron emits (creates) phonons in the vicinity of the electron's starting point, in order to reduce its energy to near the bottom of the band, and then forms a polaron (see the panel at $t=6$ ). One of the most important properties of the polaron is an increased inertial mass, for the reason that some phonons have to travel with the particle (as indicated by the enhanced on-site EP correlations). At the same time we observe a "backscattered" current [14], evolving to a left moving polaron. When the right-moving polaron reaches the wall at site 12 it will be partly reflected. More importantly, the additional local EP interaction $\varepsilon_{p, 12}$ renormalises the on-site adiabatic potential at site 12 , i.e. leads to a local polaron level shift that softens the barrier $\Delta_{12}=2$. As a result a vibrationmediated tunnelling of the particle takes place, whereby some phonons are stripped at the barrier and are recollected by the transferred particle afterwards (cf. the snapshots from $t=10$ to 14 ). Finally, the particle is reflected at the boundary and moves to the left passing the barrier again. Note that during the whole run time uncorrelated phonon excitations remain in the system, especially near the injection point. In our opinion this example impressively demonstrates that our approach can be used to monitor the complicated multiple time-scale dynamics of quasiparticle transport in finite quantum structures.

\section{Conclusion}

To summarise, in this work we compared various numerical approaches to the dynamics of complex quantum systems: expansion into eigenstates, iterative Crank-Nicholson and Chebyshev schemes, as well as semiclassical WignerMoyal and quantum tomography methods. The different methods have been applied to several physical systems and problems, ranging from motion in a simple double-well toy model to questions of current interest such as electron transport in disordered graphene nanoribbons or polaron motion in a finite quantum structure.

The Wigner-Moyal approach, evaluated in first order of the iteration series, essentially reproduces the quantum dynamics. Nevertheless important quantum interference effects, appearing in the exact solution, are missed. The successful application of the quantum tomogram to the time evolution of quantum systems crucially depends on a suitable sampling algorithm for the coordinates at which the potential is evaluated. If those are sampled according to trajectories of classically propagated particles, the result for the quantum dynamics is rather poor and fails to describe tunnelling and anharmonicity effects correctly. While the moderate numerical costs of the first order Wigner-Moyal approach seem appealing for a possible application to more complex systems, the computational resources required by the quantum tomographic approach are high in general.

On the side of the exact techniques, the Chebyshev approach largely outperforms the standard Crank-Nicholson scheme, both in computation speed (usable time step) and treatable system sizes (only matrix-vector multiplications 
were required). We conclude that the Chebyshev approach represents a very efficient and reliable tool to determine the quantum dynamics even for rather complex interacting many-particle systems.

\section{References}

[1] E. Abrahams, P. W. Anderson, D. C. Licciardello, and T. V. Ramakrishnan. Phys. Rev. Lett., 42:673, 1979.

[2] A. S. Arkhipov and Y. E. Lozovik. Phys. Lett. A, 319:217, 2003.

[3] A. S. Arkhipov, Y. E. Lozovik, and V. I. Man'ko. Phys. Lett. A, 328:419, 2004.

[4] R. Chen and H. Guo. Comp. Phys. Comm., 119:19-31, 1999.

[5] J. K. Cullum and R. A. Willoughby. Lanczos Algorithms for Large Symmetric Eigenvalue Computations, Birkhäuser, Boston, 1985.

[6] S. R. Deans. The Radon transform and some of its applications. John Wiley \& Sons, New York, 1983.

[7] H. Fehske, A. Alvermann, and G. Wellein. In S. Wagner, M. Steinmetz, A. Bode, and M. Brehm, editors, High Performance Computing in Science and Engineering, Garching/Munich 2007, pages 649-668. Springer-Verlag, Berlin, 2009.

[8] H. Fehske, A. Weiße, and R. Schneider, editors. Computational many-particle physics, volume 739 of Lecture Notes in Physics. Springer, Berlin Heidelberg, 2008.

[9] H. Fehske, G. Wellein, J. Loos, and A. R. Bishop. Phys. Rev. B, 77:085117, 2008.

10] V. S. Filinov. Nucl. Phys. B, 271:717, 1986

11 V. S. Filinov. Mol. Phys., 88:1517 \& 1529, 1996

12 V. S. Filinov, Y. V. Medvedev, and V. L. Kamskyi. Mol. Phys., 85:711, 1995

[13] E. Jeckelmann and H. Fehske. Rivista del Nuovo Cimento, $30: 259,2007$

[14] L.-C. Ku and S. A. Trugman. Phys. Rev. B, 75:014307, 2007.

[15] H. W. Lee. Physics Reports, 259:147, 1995

16] S. Mancini, V. I. Man'ko, and P. Tombesi. Phys. Lett. A, 213:1, 1996 \& Found. Phys., 27:801, 1997.

[17] V. M. Pereira, F. Guinea, J. M. B. Lopes dos Santos, N. M. R. Peres, and A. H. Castro Neto. Phys. Rev. Lett., 96:036801, 2006.

[18] W. H. Press, B. P. Flannery, S. A. Teukolsky, and W. T. Vetterling. Numerical recipes. Cambridge University Press, Cambridge, 1986.

[19] G. Schubert, V. S. Filinov, K. Matyash, R. Schneider, and H. Fehske. arXiv:0810.4302, preprint 2008.

[20] H. Tal-Ezer and R. Kosloff. J. Chem. Phys., 81:3967-3971, 1984.

[21] A. Weiße and H. Fehske. Lecture Notes in Physics, 739:545, 2008.

[22] G. Wellein, H. Röder, and H. Fehske. Phys. Rev. B, 53:9666, 1996.

[23] S.-J. Xiong and Y. Xiong. Phys. Rev. B, 76:214204, 2007. 